\title{
Insights into CRAC channel gating and ion permeation
}

\author{
Patrick G Hogan ${ }^{1}$ \\ ${ }^{1}$ La Jolla Institute for Allergy \& Immunology, 9420 Athena Circle, La Jolla, CA 92037, USA \\ Cell Research (2012) 22:1105-1107. doi:10.1038/cr.2012.46; published online 27 March 2012
}

The ORAI1 store-operated calcium channel, also known as the CRAC channel, has provided many surprises. The latest is that channel gating and ion selectivity are closely intertwined.

The calcium release-activated calcium (CRAC) channel, originally defined in T lymphocytes and mast cells, is an oligomer composed of ORAI1 subunits. Its hallmarks are exquisite selectivity for $\mathrm{Ca}^{2+}$ in physiological solutions, a narrow pore as defined by permeation of small organic cations, and a very small single-channel current. The channel is recruited to ER-plasma membrane junctions and activated by STIM proteins in response to ER $\mathrm{Ca}^{2+}$ store depletion. The emerging picture of the STIM-ORAI pathway is reviewed in $[1,2]$. There has been relatively slow progress, however, in delineating the physical basis of channel gating and ion permeation. A new paper by McNally et al. [3] reports three important advances: It locates an external gate in the ORAI1 channel, implicates the gate region in ion selectivity, and in an aspect not discussed here, shows that STIMORAI stoichiometry can influence the ion selectivity of the channel.

Previous work has established that the ORAI channel is not intrinsically

Correspondence: Patrick G Hogan

Tel: +1-858-952-7175

E-mail: phogan@liai.org restricted to conducting $\mathrm{Ca}^{2+}$. $\mathrm{Na}^{+}$and other monovalent ions can permeate the channel when no divalent ions are present, but the channel is selective for $\mathrm{Ca}^{2+}$ in physiological solutions, because binding of $\mathrm{Ca}^{2+}$ at a site in the pore prevents $\mathrm{Na}^{+}$permeation. E106 in transmembrane helix 1 (TM1) of the human ORAI1 channel was implicated in $\mathrm{Ca}^{2+}$ binding by electrophysiology $[4,5]$, and the physical proximity of E106 sidechains from separate ORAI1 subunits was confirmed by disulfide crosslinking of E106C monomers [6, 7]. An unusual feature of the ORAI1 channel is that its constituent TM1 helices are in proximity and line the pore along its entire length (Figure 1). This was apparent from the block of currents by $\mathrm{Cd}^{2+}$ traversing the channel and from the disulfide crosslinking of ORAI1 monomers, when cysteine residues were introduced at positions $88,91,95,98$, or 102 in TM1 $[6,7] \cdot \mathrm{Cd}^{2+}$ block at V102C is of particularly high affinity, indicating that the V102C sidechains are close together in the permeation pathway [6]. The protein crosslinking experiments further point to limited flexibility of the helical backbone from F99-M104 in comparison to other parts of TM1, placing V102 in a narrow and relatively rigid part of the pore that could serve as an energy barrier to ion passage [7].

The new paper by McNally et al. [3] first probes entry of the organic cation 2-aminoethyl methanethiosulfonate $\left(\mathrm{MTSEA}^{+}\right)$into the E106D channel. The pairing of channel and reagent is a calculated choice. The E106D channel has a larger pore diameter than the wild-type ORAI1 channel, but is gated normally by STIM1. The MTSEA ${ }^{+}$headgroup diameter, $3.8 \AA$, is comparable to the diameter of methylammonium, which carries current through E106D channels. Methanethiosulfonate reagents are typically used to modify cysteine residues introduced at specific sites in a channel, and the modification is detected if the added substituent blocks current through the channel. Here the target is an introduced $\mathrm{G} 98 \mathrm{C}$ residue in ORAI1, and MTSEA $^{+}$rapidly blocks current when the ORAI channels have been opened by STIM1. The rate of reaction with the open channel, monitored electrophysiologically in extracellular solution lacking divalent cations, is consistent with the idea that MTSEA ${ }^{+}$ reacts as it traverses the outer part of the conductance pathway. The substantial decrease in block when MTSEA $^{+}$is applied in the presence of extracellular $\mathrm{La}^{3+}$ or $\mathrm{Ca}^{2+}$ supports this interpretation. The telling finding is that there is no block when MTSEA ${ }^{+}$is applied to the closed channel and washed away before channel opening, leading to the first important conclusion, that physiological channel activation opens a gate exterior to G98.

The authors then focus on V102, the residue positioned about one helical turn below E106. Replacement of V102 by the slightly larger nonpolar residues 


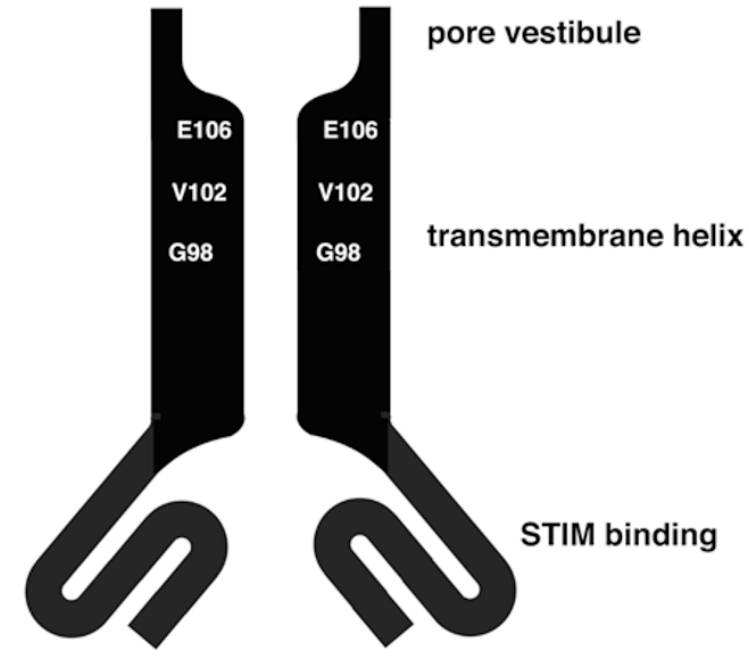

Figure 1 Schematic view of the ORAI1 channel pore, indicating the positions of residues discussed in the text. Only two of the pore-lining TM1 helices are depicted.

isoleucine, leucine, or methionine results in channels that gate normally. Replacement by the smaller residues alanine, threonine, or cysteine results in each case in a constitutively open channel whose substantial $\mathrm{Cs}^{+}$permeability, relative to the open wild-type channel, suggests a larger pore diameter. For V102C channels, an increase in pore diameter was confirmed with a series of monovalent organic cations. It would be tempting to dismiss the leaky V102A, V102T, and V102C channels as malformed. However, when the same channels are coexpressed with STIM1 and activated by $\mathrm{Ca}^{2+}$ store depletion, the $\mathrm{Cs}^{+}$permeability and where examined, the pore diameter resemble those of the wild-type channel. This brings the second important conclusion, that gating and ion selectivity of the ORAI1 channel are closely intertwined. The details can be argued, but in the very simplest scenario, V102 sidechains seal the closed channel, and gating by STIM1 moves V102 aside, exposing a narrow conductance path. Since ions traversing this path were not solvated by V102 sidechains in the wild-type channel, it is not surprising that the re- placements at V102 have little effect on the ion selectivity of STIM1-activated channels.

Along with the difference in minimal pore diameter, the current through V102C channels activated by STIM1 exhibits greater selectivity for $\mathrm{Ca}^{2+}$ than the constitutive current. Since $\mathrm{Ca}^{2+}$ selectivity typically reflects the efficient block of monovalent ion current by $\mathrm{Ca}^{2+}$, this finding suggests that gating of the V102C channel by STIM also causes a repositioning of the E106 sidechains.

The idea of a gate at V102 is readily harmonized with data on STIM-ORAI binding interactions. Initial ORAI recruitment to ER-plasma membrane junctions is through the interaction of STIM with a C-terminal segment of ORAI. Channel gating is likely to involve a second STIM binding site in the ORAI N-terminus, between residues 65 and $91[8,9]$. This second site is contiguous with TM1 in the primary sequence, and it is an attractive notion that STIM binding initiates a movement of TM1 that propagates to V102.

Zhang et al. [10] have recently proposed the alternative view that ORAI channel gating depends on a flexing of TM1 helices at G98 that widens the inner mouth of the pore. Their point of departure is the reversible block of CRAC current through R91C channels upon oxidative crosslinking of neighboring $\mathrm{R} 91 \mathrm{C}$ residues. This recalls the partial block of the open R91C channel by $\mathrm{Cd}^{2+}[6]$, and both findings suggest that pinning TM1 helices together at their cytoplasmic ends occludes the pore. That the channel can be occluded or pinned closed in these artificial cases does not imply that the gating movement of the wild-type channel involves widening the inner mouth of the pore. However, Zhang et al. [10] make the plausible arguments that glycine is used in other channels as a hinge, that a glycine residue corresponding to G98 is conserved in ORAI channels, and that the G98A channel is unable to open. Further, the G98D channel is constitutively open, and apparently has a widened inner mouth [10]. Although the experiments do not directly demonstrate the existence of an inner gate, and the structural role of glycine in transmembrane helices is often in helix packing rather than in helix bending, the existence of a second inner gate in the wild-type channel is compatible with an outer gate at V102 if their movements during gating are coupled.

In the end, a high-resolution structure will be needed for a full understanding of gating and ion permeation in the ORAI1 channel. But it is impressive how much can be inferred from judicious application of electrophysiology and protein chemistry.

\section{Acknowledgments}

The author's work is supported by US National Institutes of Health grants AI 40127 and AI 84167.

\section{References}

1 Hogan PG, Lewis RS, Rao A. Molecular basis of calcium signaling in lymphocytes: STIM and ORAI. Annu Rev 
Immunol 2010; 28:491-533.

2 Cahalan MD. STIMulating store-operated $\mathrm{Ca}^{2+}$ entry. Nat Cell Biol 2009; 11:669-677.

3 McNally BA, Somasundaram A, Yamashita M, Prakriya M. Gated regulation of CRAC channel ion selectivity by STIM1. Nature 2012; 482:241-245.

4 Yeromin AV, Zhang SL, Jiang W, Yu Y, Safrina O, Cahalan MD. Molecular identification of the CRAC channel by altered ion selectivity in a mutant of Orai. Nature 2006; 443:226-229.

5 Prakriya M, Feske S, Gwack Y, Srikanth S, Rao A, Hogan PG. Orail is an essential pore subunit of the CRAC channel. Nature 2006; 443:230-233.

6 McNally BA, Yamashita M, Engh A, Prakriya M. Structural determinants of ion permeation in CRAC channels. Proc Natl Acad Sci USA 2009; 106:22516-22521.

7 Zhou Y, Ramachandran S, Oh-hora M, Rao A, Hogan PG. Pore architecture of the ORAI1 store-operated calcium channel. Proc Natl Acad Sci USA 2010; 107:4896-4901.

8 Park CY, Hoover PJ, Mullins FM, et al. STIM1 clusters and activates CRAC channels via direct binding of a cy- tosolic domain to Orail. Cell 2009; 136:876-890.

9 Zhou Y, Meraner P, Kwon HT, et al. Minimal requirement for store-operated calcium entry: STIM1 gates ORAI1 channels in vitro. Nat Struct Mol Biol 2010; 17:112-116.

10 Zhang SL, Yeromin AV, Hu J, Amcheslavsky A, Zheng H, Cahalan MD. Mutations in Orail transmembrane segment 1 cause STIM1-independent activation of Orail channels at glycine 98 and channel closure at arginine 91. Proc Natl Acad Sci USA 2011; 108:17838-17843. 